\title{
Validation of Dual Energy X-Ray Absorptiometry and Nuclear Magnetic Resonance in the Analysis of Body Composition in Mice
}

\author{
Kyung-Wan Baek ${ }^{1,2}$, Ji-Seok Kim', Jin Sung Park², So-Jeong Kim³, Yong-Chan Ha ${ }^{4}$, Ok-Yi Jeong ${ }^{3}$, Jun-II Yoo ${ }^{2}$ \\ 'Department of Physical Education, Gyeongsang National University, Jinju; \\ ${ }^{2}$ Department of Orthopaedic Surgery, Gyeongsang National University Hospital, Gyeongsang National University, Jinju; \\ ${ }^{3}$ Department of Convergence of Medical Science, Gyeongsang National University, Jinju; \\ ${ }^{4}$ Department of Orthopaedic Surgery, Chung-Ang University College of Medicine, Seoul, Korea
}

\section{Corresponding author}

Jun-II-Yoo

Department of Orthopaedic Surgery,

Gyeongsang National University Hospital,

79 Gangnam-ro, Jinju 52727, Korea

Tel: +82-55-750-8688

Fax: +82-55-754-0477

E-mail: furim@hanmail.net

Received: September 29, 2020

Revised: November 14, 2020

Accepted: November 16, 2020
Gyeongsang National University,

Background: As an instrument for measuring body composition in experimental animals, dual energy X-ray absorptiometry (DXA) is ideal for accuracy, cost, and measurement efficiency. However, there is too little insight into the effectiveness of the various aspects of applying DXA to experimental animals. We investigated whether to compare and verify the precision and accuracy of DXA and nuclear magnetic resonance (NMR) animal body composition analyzers. Methods: We used 30 Institution of Cancer Research mice in the study. First, in order to evaluate the reproducibility of DXA and NMR, we did repeated measurements by repositioning each mouse in anesthesia and euthanasia states. Subsequently, the accuracy of each device was evaluated by comparing the weight measured before the experiment, the weight of the tissue extracted from the mice after the experiment, and the measured DXA and NMR. In addition, when measuring the body composition of animals, we compared the time and the measurable body composition parameters and summarized the advantages and disadvantages of the 2 devices. Results: Compared to NMR, DXA had the advantage of a fast measurement of bone composition and rapid image analysis. In addition, DXA showed a higher correlation (>95\%) with fat mass, lean mass baseline than did NMR (>85\%). Conclusions: In conclusion, DXA was confirmed to have higher precision and measurement accuracy than did NMR. Therefore, DXA is an effective method for evaluating the body composition of experimental animals.

Key Words: Absorptiometry, photon · Body composition - Magnetic resonance spectroscopy $\cdot$ Mice

\section{INTRODUCTION}

Animal clinical trials are conducted in various ways in order to verify the effects Mineral Research

This is an Open Access article distributed under the terms of the Creative Commons Attribution Non-Commercial License (https://creativecommons.org/licenses/by-nc/4.0/) which permits unrestricted non-commercial use, distribution, and reproduction in any medium, provided the original work is properly cited. of various physical activities of exercise on health as well as for research on the treatment of diseases and the efficacy of food. In addition, animal clinical trials are a procedure that verifies the safety of new drugs or treatments before humans are exposed to new molecular entities.[1,2] Of the many indicators used to confirm the condition in animal clinical trials, the most basic ones are body composition, such as weight, fat mass (FM), and bone mineral content (BMC). Therefore, there 
are various experimental methods for measuring these body components.

Methods of measuring body composition in human clinical practice include underwater weighing [3] and air displacement plethysmography (also known as Bod Pod),[4] which are very accurate. Underwater weighing are methods of assessing body FM levels using the difference in weight in air and water. Body fat provides more buoyancy, so obese person (on a relatively basis) weigh less than lean person. $[5,6]$ The air displacement plethysmography uses the same principles as underwater weighing, but relies on air displacement to assess body composition.[4] However, they require a lot of space, are hassle in measurement, and are expensive. In addition, skinfold thickness measurements [7] and bioelectric impedance analysis [8] have a disadvantage, in that the errors caused by the measuring operators and measurement timing are very large. Therefore, dual energy X-ray absorptiometry (DXA) is recognized as the "gold standard" because of its accuracy, cost, and measurement efficiency.[9-11] In measuring human body composition, nuclear magnetic resonance (NMR) is based on the measurement of cross-sectional areas calculated at different levels of the body based on images of body tissues. In general, NMR is also known to measure body composition as accurate as DXA.[12,13]

In animal studies, the underwater weighing used in human clinical studies has limitations in measuring bone density and region of interest (ROI), because it can only distinguish between body FM and lean body mass (LM). In addition, in the case of air displacement plethysmography, it is difficult to realize the sensitivity to measure the air displacement of an animal because of the sensitivity to measure the air displacement. Skinfold thickness method cannot be applied to animals because it relies on the calculation formula from human body data accumulated through various studies. Since electrical bioimpedance analyses focus on people's convenience rather than accuracy, they have little value to apply to animals. Computed tomography (CT) can also be considered as a method of measuring animal body composition. However, the radiation exposure of the experimenter is more than 150-fold lager than that of DXA, and there is no advantage compared to DXA or NMR in terms of cost to use it to measure animal body composition.[14] Therefore, the practical methods for measuring the body composition of animals is the measurement us- ing DXA and NMR. However, NMR is costly and impractical for most applications, resulting in measurement errors due to the problem of choosing a representative site for measurement.[15]

Like human body composition measuring, animal body composition measuring must be able to identify factors such as body weight, body fat, BMC, and the cost and accuracy of the measurement must also be considered. Considering these factors, DXA and NMR may be candidates for the most suitable equipment for measuring body composition in animals. Nevertheless, studies to select the "gold standard" equipment for measuring body composition in animals by DXA and NMR are insufficient. In particular, the only study comparing the accuracy of DXA and NMR in mice focused on comparing of body FM and LM, but bone mineral density (BMD) and BMC were not analyzed.[16] Also, as a disadvantage of DXA, the problem of measurement accuracy due to anesthesia is mentioned.[16] However, in the actual study, it was judged that if the experimental animals were well anesthetized and the movement was minimized, the effect on accuracy would be negligible. In this study, the accuracy of DXA was measured in measuring BMD and BMC (NMR could not be compared because bone and mineral do not contribute to the NMR signal),[17] and the accuracy of DXA and NMR body composition measurement was compared according to anesthesia conditions (anesthesia, euthanasia).

\section{METHODS}

\section{Experiment outline}

A total of 30 mice were used in the experiment (10 mice/ group), and a body weight target was set for each mouse group to start the experiment. In the breeding stage before the experiment, 12 candidate mice were reared for each group, and the body weight was monitored through an electronic scale. When the target body weight was reached, it was used in the experiment, and 2 animals were excluded per group. The main purpose of this study is to compare the accuracy of body composition values measured by DXA and NMR. Therefore, the FM and LM measurements of DXA and NMR were compared with the weight of the dissected tissue (autopsy). In addition, the reproducibility of each equipment was confirmed from the coefficient of variation (CV) of the measurement item. 


\section{JBM}

\section{Animals}

We purchased male Institution of Cancer Research mice $(n=30)$ from Orient Bio (Seongnam, Korea). We provided all animals with ad libitum access to both water and normal diet feed. We kept the mice in a room maintained at $23^{\circ} \mathrm{C} \pm 3^{\circ} \mathrm{C}$ with $50 \% \pm 20 \%$ relative humidity and a 12 -hr light/12-hr dark cycle. After the 1-week adaptation period, we divided the mice into a low-weight group $(10 \mathrm{~g}-20 \mathrm{~g}$, $\mathrm{n}=10$ ), mid-weight group (20 g-30 g, $\mathrm{n}=10)$, and highweight group ( $>30 \mathrm{~g}, \mathrm{n}=10$ ). The target body weight of the low-weight group was 10 to $20 \mathrm{~g}$, that of weight of the mid-weight group was 20 to $30 \mathrm{~g}$, and that of the highweight group was more than $30 \mathrm{~g}$. We sacrificed all mice in each group when they reached the target body weight.

\section{Procedure}

When measuring DXA (iNSiGHT VET DXA; OsteoSys, Seoul, Korea) or NMR (EchoMRI ${ }^{\mathrm{TM}}$-700; EchoMRI, Houston, TX, USA), there are a total of 2 measuring conditions for animals, which are defined as euthanasia with repositioning (RE), anesthesia with repositioning (RA). In the initial preparation of all animals, the weight is measured once using an electronics scale. In addition, this value is defined as a reference value of weight for accuracy verification by measuring it once more on the day of the experiment. When measuring DXA, the mouse's abdomen was positioned downward in the center of the measuring plate, and the limbs were placed in a lightly straight outward direction. During repositioning, the mouse was completely separated from the measuring plate, and then the mouse was positioned in the same way as before according to the scale printed on the measuring plate. In NMR measurement, the mouse is pushed all the way into the cylinder and a fixing device is inserted, but there is a margin to prevent the mouse from being compressed. When repositioning, after removing the mouse from the cylinder, positioning was performed in the same way before.

\section{DXA measurement}

On the day of the experiment, we measured 7 times per all mice under both anesthesia and euthanasia conditions. We repositioned animals at the initial 3 measurements, and posture at the 3rd measurement was maintained for the next 4 measurements. After relocating the animals in the initial 3 measurements, the posture was maintained dur- ing subsequent measurements. The time required for one measurement of DXA equipment was measured with a stopwatch. The time required was defined as the time until the operation was completed after pressing the measurement start button of the equipment software. We used the DXA equipment to collect FM, LM) body weight, BMD, $B M C$, and fat percentage of the entire body range. In addition, we collected left femur BMC (IfBMC) and femur BMC (fBMC) by designating the femur region as a local ROI value. Finally, we collected images provided by DXA. All DXA image shooting was done using "mouse mode" in Insight software (Version 1.0.6; OsteoSys, Seoul, Korea). The X-ray tube settings in "mouse mode" were $60 \mathrm{kV} / 0.8 \mathrm{~mA}$ and 80 $\mathrm{kV} / 0.8 \mathrm{~mA}$ at low energy and high energy, respectively.

\section{NMR measurement}

On the day of the experiment, we measured all mice 3 times each by NMR after anesthesia. We repositioned the animals at every measurement. On the day of the experiment, we measured all mice 3 times each by NMR after euthanasia. We repositioned the animals at every measurement. We measured the time required for one measurement with the NMR equipment with a stopwatch. The time required was defined as the time until the operation was completed after pressing the measurement start button of the equipment software. We collected FM, LM, and body water over the entire body range using NMR.

\section{Autopsy}

After we completed the measurement with all equipment, the mouse was autopsied, and after we extracted the main tissue, the mouse was weighed by using an electronic scales (Sartorius Entris ${ }^{\circledR} 124-1$; ; Sartorius Corp., Göttingen, Germany). We combined the weights of kidney adipose tissue, epididymal adipose tissue, intestine adipose tissue, and subcutaneous fat, and defined it as a reference value of FM for accuracy verification. The sum of the measurements of left femur and right femur was defined as femur mass and as the reference value of fBMC for accuracy verification. We selected one mouse from each group (lowweight, moderate-weight, and high-weight groups) and measured the time required for autopsy, which was defined as the time from the moment of taking the action necessary to autopsy the euthanasia animal to the completion of weighing for all items. 


\section{Statistical analysis}

To evaluate the precision, we calculated the $\mathrm{CV}$ of each item in the repeated measurement data. The mean \pm standard deviation (SD) of the $\mathrm{CV}$ values for each measurement is summarized according to the mouse condition. In order to evaluate the accuracy, we summarized average residual values for each measurement $r^{2}$ value of the fitting linear model together with reference values. In addition, we did independent 2 sample $t$-tests with equipment measurement and reference values to find the statistical significance of the mean values. Statistical analysis of the fitting linear model and independent 2 sample $t$-test used $\mathrm{R}$ software (version 3.6.1; The R Foundation for Statistical Computing, Vienna, Austria).

\section{RESULTS}

\section{Precision}

We derived the $\mathrm{CV}$ of body composition measurement results according to the measuring conditions of all mice

Table 1. Results of dual energy X-ray absorptiometry and nuclear magnetic resonance according to repeated measures

\begin{tabular}{lcc}
\hline & DXA $(n=30)$ & NMR $(n=30)$ \\
\hline FM (CV) & & \\
RE & $3.88 \pm 2.53$ & $17.00 \pm 12.91$ \\
RA & $5.05 \pm 3.58$ & $14.96 \pm 10.46$ \\
LM (CV) & \\
RE & $0.38 \pm 0.18$ & $3.91 \pm 2.39$ \\
RA & $0.43 \pm 0.25$ & $2.75 \pm 1.70$ \\
Body weight (CV) & & \\
RE & $0.27 \pm 0.15$ & NA \\
RA & $0.41 \pm 0.34$ & NA \\
Body water (CV) & & \\
RE & NA & $26.66 \pm 43.77$ \\
RA & NA & $13.65 \pm 29.73$ \\
BMC (CV) & & \\
RE & $2.36 \pm 1.44$ & NA \\
RA & $2.90 \pm 1.85$ & NA \\
IfBMC (CV) & & NA \\
RE & $6.38 \pm 3.73$ & NA \\
RA & $7.70 \pm 5.95$ & \\
\hline
\end{tabular}

The data is presented as the mean \pm standard deviation.

DXA, dual energy $X$-ray absorptiometry; NMR, nuclear magnetic resonance; $F M$, fat mass; $C V$, coefficient of variation; $R E$, euthanasia with repositioning; $R A$, anesthesia with repositioning; $L M$, lean mass; $B M C$, bone mineral content; IfBMC, left femur bone mineral content; NA, not applicable.
(Table 1). For the analysis of body composition elements, we derived the average value by classifying the repositioning condition (RE, RA) for each equipment for precision analysis. For both RE (3.88 \pm 2.53 vs. $17.00 \pm 12.91)$ and $R A$ (5.05 \pm 3.58 vs. $14.96 \pm 10.46)$, FM had lower CV than NMR. Also, in both RE (0.38 \pm 0.18 vs. $3.91 \pm 2.39)$ and $R A(0.43 \pm$ 0.25 vs. $2.75 \pm 1.70$ ) in LM, DXA had lower CV than NRM. We measured body weight, BMC, and IfBMC in DXA but not in NMR. In DXA, the CV values of body weight, BMC, and IfBMC were lower in RE than in RA, which was consistent with the direction of FM and LM (FM>LM). We measured CV of body water only in NMR, but it was lower in RA than in RE as in FM and LM. In particular, the CV value in the RE $(26.66 \pm 43.77)$ condition of body water was the highest regardless of all measurement tools, measurement conditions, or measurement items.

In order to confirm the error of measurement according to the body weight, the weight was divided in the 3 grades; the measured CV is shown in Table 2. For DXA, we confirmed that the $\mathrm{CV}$ value in all items decreased as the body weight increased, regardless of the measurement conditions. However, for NMR, the CV value of the low-weight group was high in both RE and RA. In addition, NMR showed that in all items except for FM under RA, the CV values were high

Table 2. Results of dual energy X-ray absorptiometry and nuclear magnetic resonance according to repeated measurements classified by body weight

\begin{tabular}{|c|c|c|c|c|}
\hline & \multicolumn{2}{|c|}{ RE } & \multicolumn{2}{|c|}{ RA } \\
\hline & DXA & NMR & DXA & NMR \\
\hline \multicolumn{5}{|c|}{ Low-weight $(n=10)$} \\
\hline FM (CV) & $5.63 \pm 2.90$ & $27.03 \pm 14.69$ & $6.92 \pm 4.72$ & $19.69 \pm 13.08$ \\
\hline LM (CV) & $0.36 \pm 0.21$ & $6.11 \pm 2.17$ & $0.44 \pm 0.32$ & $2.75 \pm 1.93$ \\
\hline $\mathrm{BMC}(\mathrm{CV})$ & $3.51 \pm 1.56$ & NA & $3.51 \pm 1.56$ & NA \\
\hline \multicolumn{5}{|c|}{ Mid-weight $(n=10)$} \\
\hline FM (CV) & $3.47 \pm 2.01$ & $6.11 \pm 2.17$ & $5.48 \pm 2.58$ & $15.28 \pm 8.69$ \\
\hline LM (CV) & $0.41 \pm 0.17$ & $2.51 \pm 1.49$ & $0.42 \pm 0.25$ & $2.75 \pm 1.93$ \\
\hline $\mathrm{BMC}(\mathrm{CV})$ & $1.70 \pm 1.08$ & NA & $2.47 \pm 2.11$ & NA \\
\hline \multicolumn{5}{|c|}{ High-weight $(n=10)$} \\
\hline FM (CV) & $2.54 \pm 1.61$ & $8.29 \pm 4.29$ & $3.17 \pm 2.58$ & $8.29 \pm 4.29$ \\
\hline LM (CV) & $0.38 \pm 0.15$ & $2.91 \pm 1.49$ & $0.44 \pm 0.21$ & $2.66 \pm 1.70$ \\
\hline BMC (CV) & $1.86 \pm 0.89$ & NA & $2.47 \pm 2.11$ & NA \\
\hline
\end{tabular}

The data is presented as the mean \pm standard deviation.

$R E$, euthanasia with repositioning; $R A$, anesthesia with repositioning; DXA, dual energy X-ray absorptiometry; NMR, nuclear magnetic resonance; FM, fat mass; CV, coefficient of variation; LM, lean mass; BMC, bone mineral content; NA, not applicable. 

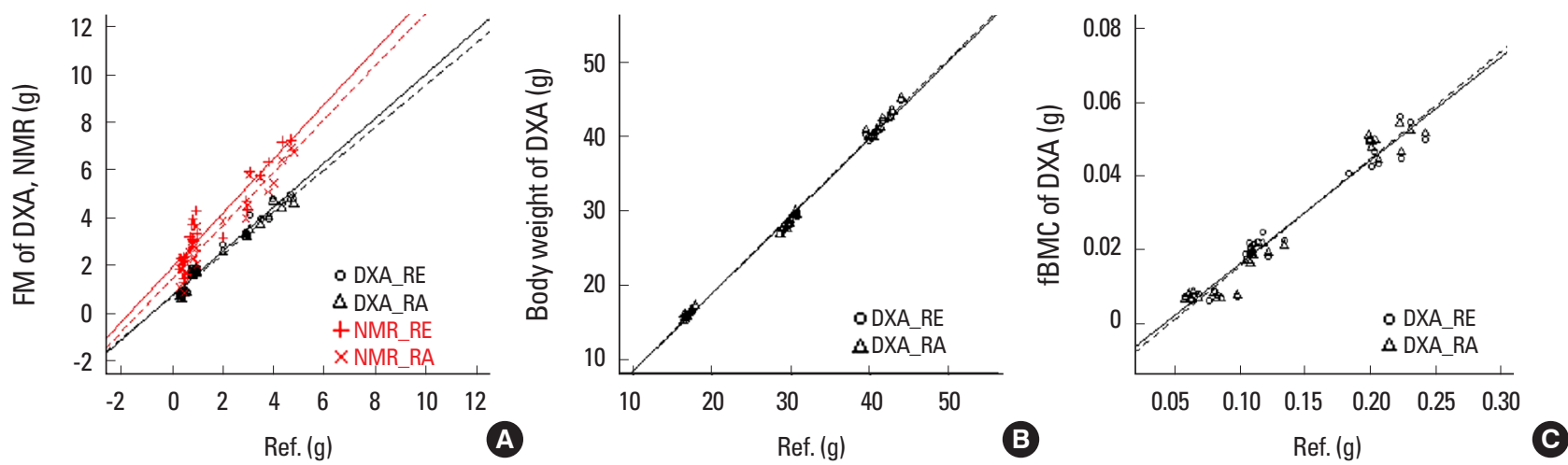

Fig. 1. Scatter plot of correlations between measurement results and reference values of dual energy X-ray absorptiometry (DXA) or nuclear magnetic resonance (NMR). RE, euthanasia with repositioning; RA, anesthesia with repositioning; FM, fat mass; fBMC, femur bone mineral content.

Table 3. Correlation between measurement results and reference values of dual energy $\mathrm{X}$-ray absorptiometry and nuclear magnetic resonance

\begin{tabular}{lccc}
\hline & DXA $(n=30)$ & NMR $(n=30)$ & Reference $^{b)}$ \\
\hline FM & & & $1.63 \pm 1.51$ \\
RE & $0.61(0.959)$ & $2.08(0.851)^{\mathrm{a})}$ & \\
RA & $0.49(0.965)$ & $1.63(0.916)^{\mathrm{a})}$ & \\
Body weight & & & $29.36 \pm 10.16$ \\
RE & $-0.80(0.996)$ & NA & \\
RA & $-0.60(0.996)$ & NA & \\
IfBMC & & & $0.133 \pm 0.060$ \\
RE & $-0.108(0.954)$ & NA & \\
RA & $-0.108(0.961)$ & NA & \\
\hline
\end{tabular}

The data is presented as mean signed difference $\left(r^{2}\right)$ or mean \pm standard deviation.

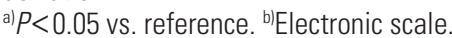

DXA, dual energy $X$-ray absorptiometry; NMR, nuclear magnetic resonance; FM, fat mass; $R E$, euthanasia with repositioning; $R A$, anesthesia with repositioning; IfBMC, left femur bone mineral content; NA, not applicable.

in the order of low-weight, high-weight, and mid-weight. For LM, unlike FM, there was no tendency to increase or decrease in group average and CV.

\section{Accuracy}

We compared the measurements of each body component according to the measuring conditions with the reference values (Table 3 and Fig. 1). For indirect comparison of the measurement accuracy of DXA and NMR, we compared them against a reference value measured by an electronic scale and expressed the values as mean signed difference (MSD) and $r^{2}$ values (MSD $\left[r^{2}\right]$ ). For FM, there was no significant difference in either RE or RA of DXA compared to the reference value. However, compared to the reference val- ue, NMR had a significantly difference in both RE and RA $(P<0.05)$. NMR could not measure body weight and fBMC, because of the relatively long measurement time compared to DXA. Although comparison with NMR was not possible, DXA showed a high correlation $\left(r^{2}>0.95\right)$ with body weight.

\section{DISCUSSION}

DXA is a method of deriving body composition results using the difference between high-energy $X$-ray images and low-energy X-ray images.[18] NMR is a technology that generates high frequencies, measures the difference in signals from each tissue, and then reconstructs them by means of a computer to image them.

Compared to X-ray or CT, NMR has no radiation exposure. Its image contrast and resolution has excellent advantages for soft-tissue and brain examination,[19] but it has a disadvantage in that it takes longer to measure than does DXA. In addition, the caustic ratio is poor for measuring only body composition. Therefore, if the measurement precision and accuracy of body components are higher than those of NMR, using DXA in much more effective.

In order to reduce the errors in measuring the experimental animals, what is most important is the degree of fixation at the time of measurement. It is natural that accuracy is higher in euthanasia than in anesthesia, because under anesthesia, there is a high possibility of errors caused by differences in fine movements that depend on the degree of breathing and level of anesthesia. In general, it is known that DXA is less capable of scanning non-anesthetized animals compared to NMR.[16] This is because if an 
X-ray image is obtained while the animal is not anesthetized, the sharpness of the image decreases. For this reason, it was speculated that the accuracy of DXA results would decrease when less anesthesia was performed, but the results of our study showed much higher accuracy of DXA under both RE and RA. These results suggest that the accuracy of DXA may be higher than that NMR if mouse movement is minimized through deep anesthesia.

In previous study, it was mentioned that the measurement speed of NMR has an advantage over DXA.[16] This mention is due to the difference in the performance of the equipment, and it is difficult to assert that it is the difference in the general measurement time. In common sense, even in the image measurement method, it is thought that obtaining an image at once by emitting $X$-rays will be faster than the NMR composed of 3-dimension (3D). In particular, the measurement time of the DXA equipment used in this study was $24.7 \mathrm{sec}$ and the measurement time of the NMR equipment was $79.3 \mathrm{sec}$.

The precision of FM and LM in DXA was higher than that of NMR (Table 1). The rest of the items could not be com- pared between DXA and NMR, because DXA classifies body components by the contrast of the X-ray image (Fig. 2); so body water is included in the LM and classified. Also, because NMR is analyzed using the hydrogen spin, bone-related contents are not measured (see manufacturer's description). Although it was impossible to compare it with DXA, the standard deviation of body water was very large because of the characteristic of the NMR. Overall, it seems that neither DXA nor NMR seems to be suitable for measuring body water. Therefore, in order to measure body water, we judge that it would be more accurately measured by other methods, such as electrical bioimpedance analysis.[20]

FM had an error of less than about $1 \mathrm{~g}$ compared to the reference value and showed a correlation of more than $95 \%$. The error of NMR was $+2.08 \mathrm{~g}$, which was larger than that of DXA. Since NMR showed a correlation of up to about 92\% with the reference value, we concluded that DXA has higher estimation accuracy for the reference value than does NMR. When compared to the actual mouse body weight, DXA measured about 2.04\% to 2.96\% lower. However,

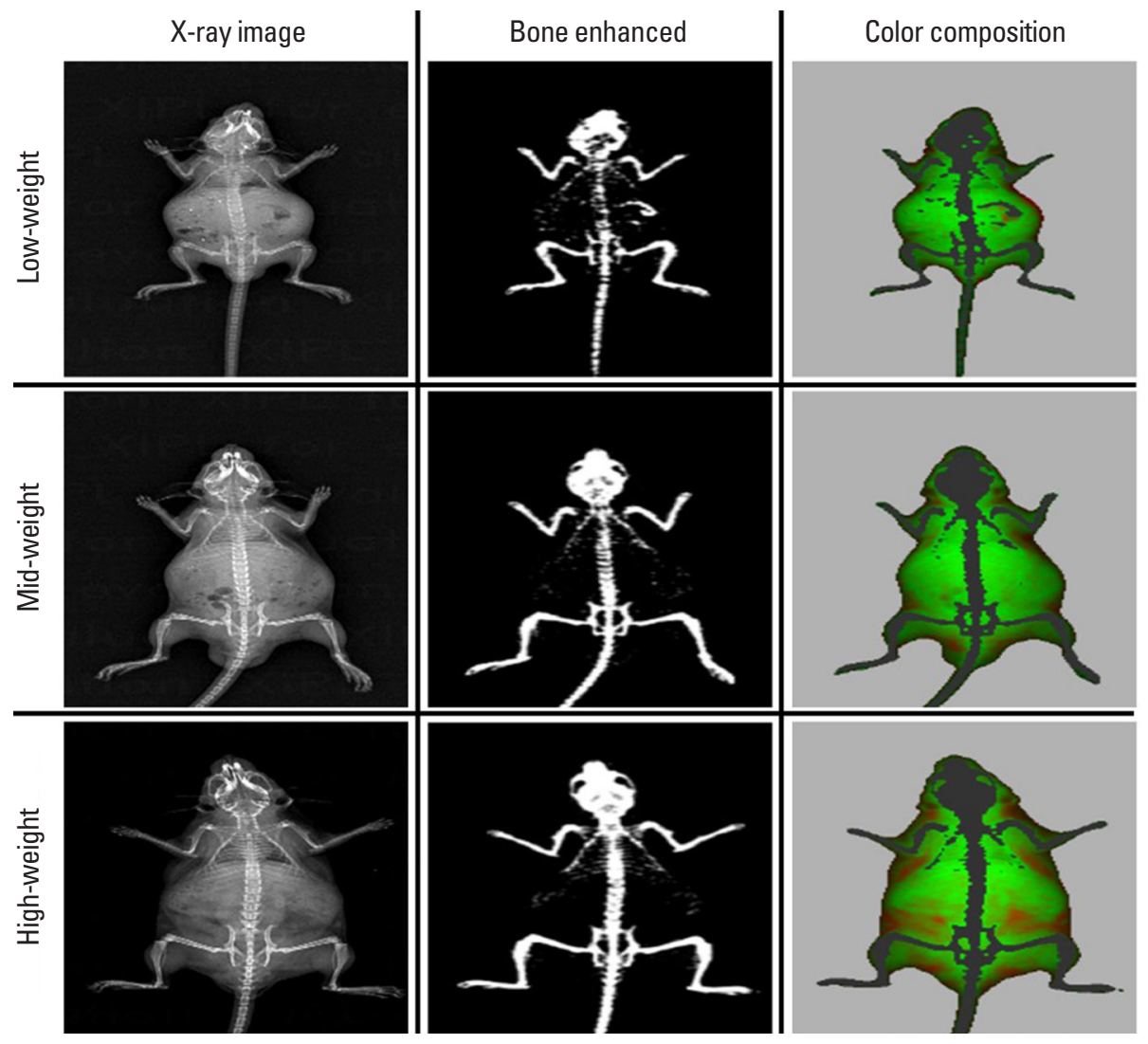

Fig. 2. Measurement images provided by dual energy X-ray absorptiometry used in this study. 
we found that the body weight difference between individuals was measured with an accuracy of $99 \%$ or more $\left(r^{2}>0.996\right)$ under all measurement conditions. The reason why the overall weight was measured was not confirmed in this experiment. However, since it is easy to obtain and there is no problem in using it with DXA, we recommend weighing the body using an electronic scale.

The $r^{2}$ value of fBMC measured in DXA showed a correlation of more than $95 \%$ with the reference value. Therefore, we judge that the difference between the bone components of the mouse individuals is relatively good. In DXA, the $\mathrm{fBMC}$ value was estimated to be under about $82 \%$ of the reference value. The reason for the large difference is that the reference value and $\mathrm{ABMC}$ measured by DXA are not actually the same component. DXA's fBMC measures the mineral component existing in the ash state by drying and completely burning the femur bones, because this study defined the reference value as the weight of the femur bone after extraction. Therefore, the value of fBMC measured by DXA may be more accurate than is the reference value. There are several studies supporting this. In a study of wet weight, dry weight, and ash weight in tibia of 3-week-old rats, the dry weight and ash weight were 53\% and $26 \%$ of the wet weight, respectively. Also, the ash weight was $47 \%$ of the dry weight.[21] In another study, the dried and ash weights were $67 \%$ and $32 \%$ of the fresh weight of the right tibia and femur of hens.[22] Another study found that the ash weight of the femur in mice was $58 \%$ of the dry weight.[23] DXA has the advantage of being able to simultaneously do both bone-related measurements and quantitative analysis of soft tissues, such as fat and lean. In addition, since X-rays used in DXA react with all substances and are attenuated, weight can be accurately calculated, and image analysis information on the shape or fat distribution of bones and organs is also provided through measurement images. However, since the X-ray image provided by DXA is a 2D cross-sectional image, the amount or accuracy of information may be deteriorated compared to the 3D structural information that can be observed by autopsying animals. Also, the DXA method has a disadvantage in that it is impossible in principle to obtain components other than bone, fat, and lean, because the components are classified using the differences in the object transmission characteristics of the $2 \mathrm{X}$-rays. However, studies have been done to obtain other components, such as moisture and protein using a statistical estimation method using DXA.[24,25]

To sum up, DXA exhibits high accuracy with little difference from the actual autopsy weight of FM. Because of this FM's accuracy, LM can also be accurately measured. However, BMD and BMC were different from the autopsied weight. This difference in weight is due to the difference in water content of bone, and BMD, BMC between individuals can be compared relatively accurately with $X$-ray images.

NMR has a longer measurement time than DXA, but has the advantage of being able to measure without anesthesia even when the animal is alive. In addition, NMR equipment can measure water in addition to fat and lean. However, since there are body tissues that cannot be measured, because they do not respond well in the NMR method, such as bone, NMR contains a fundamental error in weight estimation. In addition, there is a disadvantage in that visual information cannot be acquired, because an image of the measured animal is not provided separately.[17]

Unlike NMR, DXA can measure BMD and BMC, and it is judged to be suitable as a "gold standard" for grasping the body composition of animals because of its high precision and accuracy of measurement. In particular, if the experimental animals are deeply anesthetized to minimize movement during DXA measurement, the effect on the measurement results seem to be insignificant.

\section{DECLARATIONS}

\section{Acknowledgements}

This work was carried out with the support of "Cooperative Research Program for Agriculture Science and Technology Development (Project No. PJ014155052019)" Rural Development Administration, Republic of Korea.

\section{Ethics approval and consent to participate}

The entire experiment was done in accordance with the guidelines of the Animal Experimental Ethics Committee of the Agency for Korea National Food Cluster (approval number: KNFC-IACUC-20-001).

\section{Conflict of interest}

No potential conflict of interest relevant to this article was reported. 


\section{ORCID}

Kyung-Wan Baek

Ji-Seok Kim

Jin Sung Park

So-Jeong Kim

Yong-Chan $\mathrm{Ha}$

Ok-Yi Jeong

Jun-II Yoo https://orcid.org/0000-0002-8445-3773

https://orcid.org/0000-0002-3023-1999

https://orcid.org/0000-0002-6284-9566

https://orcid.org/0000-0001-6422-7595

https://orcid.org/0000-0002-6249-0581

https://orcid.org/0000-0001-9362-2746

https://orcid.org/0000-0002-3575-4123

\section{REFERENCES}

1. Junod SW. FDA and clinical drug trials: a short history. 2019 [cited by 2020 Aug 1]. Available from: https://www.fda. gov/media/110437/download

2. Mak IW, Evaniew N, Ghert M. Lost in translation: animal models and clinical trials in cancer treatment. Am J Transl Res 2014;6:114-8.

3. Behnke AR, Jr., Feen BG, Welham WC. The specific gravity of healthy men. Body weight divided by volume as an index of obesity. 1942. Obes Res 1995;3:295-300. http:// dx.doi.org/10.1002/j.1550-8528.1995.tb00152.x.

4. Dempster P, Aitkens $S$. A new air displacement method for the determination of human body composition. Med Sci Sports Exerc 1995;27:1692-7.

5. Siri WE. Body composition from fluid spaces and density: analysis of methods. 1961. Nutrition 1993;9:480-91; discussion, 92

6. Wilmore JH. The use of actual, predicted and constant residual volumes in the assessment of body composition by underwater weighing. Med Sci Sports Exerc 1969;1:87-90.

7. Durnin JV, Rahaman MM. The assessment of the amount of fat in the human body from measurements of skinfold thickness. Br J Nutr 1967;21:681-9. http://dx.doi.org/10. 1079/bjn19670070.

8. Segal KR, Van Loan M, Fitzgerald PI, et al. Lean body mass estimation by bioelectrical impedance analysis: a four-site cross-validation study. Am J Clin Nutr 1988;47:7-14. http:// dx.doi.org/10.1093/ajcn/47.1.7.

9. Bazzocchi A, Diano D, Ponti F, et al. Health and ageing: a cross-sectional study of body composition. Clin Nutr 2013; 32:569-78. http://dx.doi.org/10.1016/j.clnu.2012.10.004.

10. Bazzocchi A, Ponti F, Diano D, et al. Trabecular bone score in healthy ageing. Br J Radiol 2015;88:20140865. http:// dx.doi.org/10.1259/bjr.20140865.

11. Guglielmi G, Ponti F, Agostini M, et al. The role of DXA in

sarcopenia. Aging Clin Exp Res 2016;28:1047-60. http:// dx.doi.org/10.1007/s40520-016-0589-3.

12. Heymsfield SB, Wang Z, Baumgartner RN, et al. Human body composition: advances in models and methods. Annu Rev Nutr 1997;17:527-58. http://dx.doi.org/10.1146/annurev.nutr.17.1.527.

13. Shen W, Punyanitya M, Wang Z, et al. Total body skeletal muscle and adipose tissue volumes: estimation from a single abdominal cross-sectional image. J Appl Physiol (1985) 2004;97:2333-8. http://dx.doi.org/10.1152/japplphysiol.00744.2004.

14. Scholz AM, Bünger L, Kongsro J, et al. Non-invasive methods for the determination of body and carcass composition in livestock: dual-energy X-ray absorptiometry, computed tomography, magnetic resonance imaging and ultrasound: invited review. Animal 2015;9:1250-64. http:// dx.doi.org/10.1017/s1751731115000336.

15. Wells JC, Fewtrell MS. Measuring body composition. Arch Dis Child 2006;91:612-7. http://dx.doi.org/10.1136/adc. 2005.085522.

16. Halldorsdottir S, Carmody J, Boozer CN, et al. Reproducibility and accuracy of body composition assessments in mice by dual energy $x$-ray absorptiometry and time domain nuclear magnetic resonance. Int J Body Compos Res 2009;7:147-54.

17. Adam CL, Williams PA, Dalby MJ, et al. Different types of soluble fermentable dietary fibre decrease food intake, body weight gain and adiposity in young adult male rats. Nutr Metab (Lond) 2014;11:36. http://dx.doi.org/10.1186/ 1743-7075-11-36.

18. Kelly TL, Berger N, Richardson TL. DXA body composition: theory and practice. Appl Radiat Isot 1998;49:511-3. http: //dx.doi.org/10.1016/s0969-8043(97)00226-1.

19. Kolodziejski W, Klinowski J. Kinetics of cross-polarization in solid-state NMR: a guide for chemists. Chem Rev 2002; 102:613-28. http://dx.doi.org/10.1021/cr000060n.

20. Jaffrin MY, Morel H. Body fluid volumes measurements by impedance: a review of bioimpedance spectroscopy (BIS) and bioimpedance analysis (BIA) methods. Med Eng Phys 2008;30:1257-69. http://dx.doi.org/10.1016/j.medengphy. 2008.06.009.

21. Jiang SD, Jiang LS, Dai LY. Changes in bone mass, bone structure, bone biomechanical properties, and bone metabolism after spinal cord injury: a 6-month longitudinal study in growing rats. Calcif Tissue Int 2007;80:167-75. 
http://dx.doi.org/10.1007/s00223-006-0085-4.

22. Kim WK, Donalson LM, Herrera P, et al. Research note: Effects of different bone preparation methods (fresh, dry, and fat-free dry) on bone parameters and the correlations between bone breaking strength and the other bone parameters. Poult Sci 2004;83:1663-6. http://dx.doi.org/10. 1093/ps/83.10.1663.

23. Minematsu A, Yoshimura O, Yotsuji H, et al. Time course of influence by ovariectomy and calcium diet on bone properties in mice. J Jpn Phys Ther Assoc 2001;4:19-23. http:// dx.doi.org/10.1298/jjpta.4.19

24. Wilson JP, Strauss BJ, Fan B, et al. Improved 4-compartment body-composition model for a clinically accessible measure of total body protein. Am J Clin Nutr 2013;97:497-504. http://dx.doi.org/10.3945/ajcn.112.048074.

25. Speakman JR, Booles D, Butterwick R. Validation of dual energy X-ray absorptiometry (DXA) by comparison with chemical analysis of dogs and cats. Int J Obes Relat Metab Disord 2001;25:439-47. http://dx.doi.org/10.1038/sj.ijo. 0801544. 
